\title{
Evolutionary dynamics of Begomoviruses causing Papaya leaf curl disease in India
}

\author{
Aarshi Srivasatava ${ }^{1 \$}$, Vineeta Pandey ${ }^{1 \$}$, Anurag Kumar Sahu ${ }^{2}$, Dinesh Yadav $^{1}$ and \\ R.K. Gaur ${ }^{1 *}$
}

1. Department of Biotechnology, D.D.U. Gorakhpur University, Gorakhpur-273009, Uttar Pradesh, India

2. International Centre for Genetic Engineering \& Biotechnology, Aruna Asaf Ali Marg, New Delhi, Delhi - 110067, India

\section{${ }^{\$}$ Equal contribution \\ *Corrsponding author: gaurrajarshi@ hotmail.com}

\section{Abstract}

The genus begomovirus represents a group of multipartite viruses that significantly damages many agricultural crops, including papaya and drastically influence the overall production. Understanding the genetic variations, mutations and recombination of the begomovirus population infecting papaya has several important implications in alleviating substantial losses, mainly in developing countries, including India. In an attempt for a bioinformatics driven study of diversity and variability of papaya leaf curl disease in India, a total of thirtytwo (32) DNA-A and sixteen (16) betasatellite sequences were retrieved from GenBank. An uneven distribution of evolutionary divergence has been observed across the branch length, which triggered the estimated recombinational event. Interestingly, a maximum of the begomoviruses were found to be intra-species recombinants. Further genetic variability, selection pressure, and substitution rate acting on the population were estimated and found to be high enough to support the evolution of geminiviruses. Genetic divergence composition in all begomovirus datasets revealed predominance of nucleotide diversity driven by mutation. The analysis indicates that even though a significant fraction of the genetic variations might be due to recombination but, it was constantly lower than the mutation rate. Thus, the diversification of the begomovirus population is principally impelled by mutational dynamics.

Keywords: Begomovirus, Papaya leaf curl disease, Genetic variability, Phylogenetic analysis, Recombination 
37 The genus Begomovirus belongs to the family Geminiviridae consisting of 424 species, 2020 release Virus Taxonomy by the International Committee on Taxonomy of Viruses (ICTV) (Zerbini et al. 2017). A closed circular single stranded DNA encapsidated in a quasiisometric non-enveloped twinned particle of $\sim 2.8 \mathrm{~kb}$ size constitutes a virus genome (Stanley et al. 2005). The native New World begomoviruses have only bipartite genomes (DNA-A and DNA-B components) whereas, begomoviruses of the Old World constitute both monopartite and bipartite genomes (lack DNA-B and have DNA-A homolog) (Brown et al. 2012). Although in bipartite begomovirus DNA-A can replicate independently but they require DNA-B for nuclear localization. The uniqueness of monopartite is the presence of small ssDNA satellite molecules which greatly enhance the virus virulence. These molecules are alphasatellite $(\sim 1.4 \mathrm{~kb})$, betasatellite $(\sim 1.3 \mathrm{~kb})$ and a newly reported deltasatellite of approximately size of $0.7 \mathrm{~kb}$ (Brown et al. 2012; Lozano et al. 2016; Fiallo-Olivé et al. 2016). These begomoviruses are transmitted by whiteflies of the Bemisia tabaci cryptic species which has been adapted and co-evolved with the begomovirus genome (Brown et al. 2012; Marwal et al. 2021). The B. tabaci is considered as the second most important vector because of its semi-persistent mode and behavioural manipulation for transmitting plant viruses (Moreno-Delafuente et al. 2013). The genomic DNA of the monopartite genome and DNAA of the bipartite genome have corresponding genome organization consisting of six open reading frames (AV2, AV1, AC3, AC2, AC1, AC4), whereas DNA-B consists of two open reading frames $(\mathrm{BV} 1, \mathrm{BC} 1)$ which also contribute to intra as well as inter-cellular movement of viral particles within the host. Thus, together they cause systemic infection and develop typical symptoms (Nawaz-ul-Rehman et al. 2009; Hanley - Bowdoin et al. 2013). Begomoviruses commonly induce severe symptoms in their hosts, including golden mosaic, yellow mosaic and leaf curl. Devastating pathogens include members of species such as Cotton leaf curl Multan virus, African cassava mosaic virus, Chilli leaf curl virus, Bean golden mosaic virus and Tomato yellow leaf curl virus. A vast range of initial symptoms, such as green- yellow mottle/mosaic, leaf curling, interveinal yellowing, vein swelling, and yellow spots are found associated with dicotyledonous plants infected with begomoviruses. In India, papaya leaf curl disease was first identified by Thomas and Krishnaswamy in 1939, which was confirmed by Saxena and colleagues that the disease is caused by geminivirus, i.e., Papaya leaf curl virus (Saxena et al. 1998). The infection rate of disease depends on the 
69 severity rate increases with the rise in the whitefly population. The symptoms like reduction

70 in leaf size, leaf curling, vein thickening, yellow mosaic, interveinal chlorosis, and stunted

71 growth of plants with small distorted fruits or no fruits can be found consociated with papaya

72 (Shahid et al. 2013; Varun et al. 2017). However, stunting may be seen at a severe stage of

73 infection, which may eventually cause the death of infected plants.

74 Diseases associated with begomoviruses are found to be a threat to global papaya production.

75 Carica Papaya, a major tropical, sweet, large and herbaceous food crop, belongs to the order

76 Brassicales and the family Caricaceae is cultivated throughout India, and valued for its

77 medicinal and nutritional benefits (Yadav et al. 2016). The market demand for tropical fruits

78 is rapidly increasing for papaya, and now ranking third due to its benefits and importance in

79 agribusiness (Evans 2012). Despite being an important wide-ranging topical fruit grown

80 throughout the year in many portions of the country, papaya yields are degraded due to

81 infection by a number of viral diseases which are particularly important (Usha 1980; Nehra et

82 al. 2019). Fruit is significantly important due to early bearing and space conserving variety

83 and is considered as a rich source of fibres, minerals, and antioxidant nutrients. In addition, it

84 is a source of the digestive enzyme Papain, which is found as an important industrial

85 ingredient in meat tenderizing, cosmetics, beauty products, brewing and pharmaceuticals (Vij

86 and Prashar 2015; Urgessa et al. 2019). Above all, papaya acts as a metabolic activator,

87 detoxifier, homeostasis maintainer, and cell rejuvenator. The medicinal and nutritional value

88 of Papaya aids human health (Adiaha and Adiaha 2017). Furthermore, its local and

89 commercial cultivation is difficult to achieve its full potential (FAOSTAT 2019) due to the

90 incidence and emergence of a large number of plant-infecting viruses resulting in significant

91 crop loss (Nascimento et al. 2010). Majorly, leaf curl symptoms caused by papaya leaf curl

92 virus are found associated with infected papaya plants from different regions, which initiates

93 serious production losses and can act as potential inducer for transmission of viruses via

94 vector whitefly (Guo et al. 2015). The substantial diversity of begomoviruses affecting

95 papaya in India includes several reports such as papaya leaf curl virus (PaLCuV) found

96 infecting papaya for the first time described in 1939 in India. Since then, papaya leaf curl

97 virus has been found as an important repressor for papaya cultivation. In spite of being

98 economically important tropical fruit, little attention has been paid to assess the genetic

99 diversity at the molecular level for papaya leaf curl virus causing leaf curl disease in papaya

100 (Fougat et al. 2015). 
101 This article provides an insight into genomic components with its satellite molecules

102 associated with the begomovirus causing leaf curl disease, its diversity and evolution patterns 103 using bioinformatics approach.

\section{Materials and methods}

\section{Sequence retrieval and Sequence alignment}

Genome sequences of begomoviruses infecting papaya reported till June 2021 were retrieved from GenBank (www.ncbi.nlm.nih.gov) and their distributions along with its sub-viral satellite across India were depicted. (Table 1; Fig1). Three sequence datasets for whole genome sequences of 32 DNA-A segment, its six ORFs and 16 associated betasatellite were prepared for further analysis. Only the sequences containing distinct nanomer "TAATATT" were considered for the present studies. Each specific datasets were aligned through multiple sequence alignments algorithm with Clustal W, using MEGA X software (Kumar et al. 2018).

\section{Phylogenetic analysis}

Evolutionary divergence helps to observe sequence diversity among the virus isolates. The wide range of distribution helps in understanding the viruses' origin, dispersion, development and etiology of diseases. Based on lowest BIC value, the best nucleotide substitution model were chosen using model test ultimately constructed a phylogenetic tree utilizing 1,000 bootstrap replicates for maximum likelihood (ML) algorithm embed in MEGA X software (Kumar et al. 2018). Different transversion and transitional substitutions rate together with transition/transversion bias (R) were also calculated using MEGA X program. (Kumar et al. 2018).

\section{Detection of Recombination}

The aligned nucleotide sequences of reference begomovirus were used to analyse the evidence of recombination by utilizing recombination detection program (v.4.2). Different algorithms of RDP 4.2 software such as RDP, BOOTSCAN, CHIMAERA, GENECONV, MAXCHI, 3SEQ and SISCAN (Martin et al. 2015) were used to detect a potential recombination event by predicting foremost and rearmost breakpoints, parental isolates and origin of viruses. For analysis, towards 0.05 highest acceptable Bonferroni corrected p-value 
and default detection thresholds datasets were subjected. To escape false-positive results, out of seven at least three algorithms were regarded appropriate to detect recombination events.

\section{Population structure and Coalescent analysis}

To investigate the genetic variability and diversity in the virus population several parameters embed in DnaSP (v. 6.12) software used (Rozas et al. 2017). The aligned sequence datasets were analysed for, total number of segregating sites (s), the average number of nucleotide differences between sequences $(\mathrm{k})$, total number of mutations $(\eta)$, nucleotide diversity $(\pi)$, additionally, Watterson's estimate of the population mutation rate based on the total number of segregation sites $(\theta-w)$ and the total number of mutations $(\theta-\eta)$ were also calculated along with a number of haplotypes (h), and haplotype diversity (Hd) (Lima et al. 2017).

Furthermore, an implemented method in DnaSP (v.6.12.) software i.e., Neutrality test, are executed to calculate the hypothesis of selection pressure occurring in population. Therefore, sequence datasets separated by different geographical location are tested by employing tests such as Tajima's $D$, Fu and Li's $D^{*}$ for identifying the difference between the total number of mutations and the number of singletons, and $\mathrm{Fu}$ and $\mathrm{Li}$ 's $F^{*}$ for identifying the difference between the average number of nucleotide differences between paired sequences and the number of singletons (Rozas et al. 2017).

Consequently, the mean substitution rate and mutational bias in the sequence datasets of begomoviruses were determined by using the Bayesian Markov Chain Monte Carlo (MCMC) parameter of BEAST (v.1.10) (Suchard et al. 2018). Coalescent constant demographic models and Best-fit molecular clock were detected employing BEAST and resultant were operated under Tracer program (v 1.5) to achieve effective sample size (Rambaut et al. 2018). MCMC chain used $10 \%$ burn-in value with $10^{7}$ run lengths to provide $95 \%$ highest posterior density (HPD) interval for determining statistical uncertainty.

\section{Results}

Since 1990s, humans adapting to modernisation and India's changing climatic condition have been found promoting factors in the emergence and evolution of many begomovirus diseases. Frequent recombination and mutation such as nucleotide substitution are apparent as topological incongruence in evolutionary events which depend upon type of virus and host plant (Varun and Saxena 2018). The occurrence of leaf curl disease in papaya was observed 
which allowed to device a molecular perceptive for understanding the DNA polymorphism of begomoviruses.

\section{Phylogenetic analysis and Detection of Substitution bias}

Using MEGA X program, the evolutionary history for sequence datasets DNA-A and associated betasatellite were calculated using the ML Tree based on the best fit nucleotide substitution model i.e., (TN93+G) for DNA-A, its ORFs and (TN93+G+I) for betasatellite computing with CLUSTAL W pairwise alignment. The four distant lineages were observed with 1000 bootstrap support and grouped as (ChiLCV I a \& b), (PaLCuV II), (PaYLCuV III), (PaLCrV IV) (Fig2 a). The lineages comprise the isolates collected from different smaller and larger geographic locations (Fig1). The branch length among population suggests the level of differentiation within them. In addition, well-defined clusters are observed in case of betasatellite depicting four clades distinct grouped as (ToLCB I), (ChiLCB II), (ToLCB III), (CroYVMB IV) (Fig2 b). Since, longer branches are reported to be associated with well supported recombination events though short branches also support the recombination (Lima et al. 2017). Accordingly, the mean branch lengths found in the present study might be contributing to recombinational events. Nevertheless, the results also indicate that begomoviruses infecting Carica papaya were not restricted to any solitary geographical region of India.

Moreover, different rate of transitional, transversion substitutions and transition/transversion bias (R) were estimated for the begomoviruses causing papaya leaf curl disease (Table 2). The rate of transitional substitutions ranged from 9.77 to 15.6, and transversional substitutions ranged from 5.41 to 7.71 while transition/transversion bias $(\mathrm{R})$ of 0.97 was observed for DNA-A. Further, its six ORF's showed variable values based on gene nature for different substitution rate. The transitional rate was maximum for $\mathrm{C} 4$ gene while Rep gene showed minimum value. Similarly, maximum transversional substitutions rate was observed for REn gene while pre-CP gene showed minimum value. The highest and lowest transition/transversion bias (R) was observed for TrAP and C4 gene respectively. Similarly, for betasatellite the range obtained for transitional and transversional substitutions was 8.49 to 14.62 and 5.64 to 9.26 respectively while, the transition/transversion bias (R) was 0.82 . However, the estimation might support the contribution of mutation for nucleotide polymorphisms in a population. 


\section{Detection of Recombination}

193 Further, the tree- like phylogenetic divergence obtained for sequence datasets directed us to 194 detect the occurrence of non-tree-like evolution within populations to explain the potential of 195 recombination events in aligned sequences. For analysis, different parameters were used for 196 determining the shared overlapping intra- and inter-specific recombination events distributed 197 throughout the genome with different parental combinations. Thirty-two putative 198 recombination events were observed for (DNA-A) datasets and sixteen putative 199 recombination breakpoints were identified for betasatellites (Table 3a). However, intra200 specific recombination among different reference sequences were predominately observed in $201 \mathrm{AC} 1, \mathrm{AV} 1$ and $\mathrm{AC} 2$ rich genome region while $\mathrm{AV} 2, \mathrm{AC} 3, \mathrm{C} 4$ genome region showed 202 minimum effect. This substantiate the possibility of putative recombination breakpoints 203 among ORF's of theses DNAs (Table 3b). Moreover, recombination events distributed 204 predominantly in the $\beta \mathrm{C} 1$ genome region of betasatellites supports the prevalence of 205 recombination that is involved in virus movement by suppressing host antiviral silencing 206 gene (Kumar et al. 2015). Further, relevant recombination events were obtained by selecting 207 at least three or more methods which minimize incompetent outcomes. Thus, significant 208 amounts of genetic variation were supported by maximum putative recombinational events 209 among sequence datasets.

\section{Population Structure and Coalescent analysis}

211 An evolutionary scenario signifies the role of nucleotide substitution along with 212 recombination in gaining genetic variation and evolution among begomoviruses (Mishra et al. 213 2020). The mean substitution rate among sequences datasets of DNA-A were $2.80 \times 10^{-4}$ sub

214 site $^{-1}$ year $^{-1}$ (DNA-A, 95\% highest posterior density (HPD) interval ranging from $3.541 \times 10^{-5}$ 215 to $9.747 \times 10^{-4}$ ), which is comparatively higher when compared with the range of nucleotide 216 substitution rate of RNA viruses thus, suggesting that though geminivirus evolve at 217 analogous rate as many RNA viruses reported so far (Jenkins et al. 2002; Duffy and Holmes 218 2009; Kumar et al. 2015) but importantly the high substitution frequency detected here shows 219 short term mutational phenomenon acting on population rather than long term substitution 220 rate. To justify above, high rate of nucleotide substitutions was also detected superficial in the 221 three gene datasets i.e., pre-CP, REn, Rep (Table 4). In addition, $5.14 \times 10^{-5}$ sub site $^{-1}$ year $^{-1}$ $222\left(\beta, 95 \%\right.$ HPD interval ranging from $7.157 \times 10^{-7}$ to $1.56 \times 10^{-4)}$ substitution rate was detected 223 for betasatellites. Conversely, relaxed molecular clock is used as prior to get the suitable 
224 value of mean substitution rate and the detected high substitution value are questionable to be

225 caused by strong positive selection (Duffy and Holmes 2008, 2009).

Since, selection pressure acting for genetic variation was also effectuated by codon degeneracy, therefore mutational selection pressure within three nucleotide codon positions i.e., $\mathrm{CP} 1, \mathrm{CP} 2, \mathrm{CP} 3$ respectively, were appraised and found higher at codon position $\mathrm{C} 3$ for DNA-A and among ORFs highest codon degeneracy were found in CP gene at codon position C3 as compared to other genes. Similarly, for betasatellites chief mutation rate were found at codon position C3 (Table 4).

Moreover, to determine the degree of genetic variability, demography structure analysis was estimated (Table 5). However, the analysis revealed for DNA-A, number of polymorphic sites (s) were 1737 with 2720 number of mutation $(\eta)$ having nucleotide diversity $(\pi)$ of $(\pi=0.2)$ which were found greater on comparison with CLCuMulV (RV-1) having a low degree of genetic variability $(\pi>0.08)$ (Mishra et al. 2020). Similarly, variability was seen in the ORFs of the DNA- A, the significant contributor of high degree of genetic divergence was verified by the non-randomness of nucleotide variability throughout genome region which were performed effectively by $\mathrm{C} 4$, Rep and REn gene depicting highest nucleotide diversity $(\pi)$ value (Table 5). Simultaneously, for betasatellite a number of polymorphic sites (s) were 834 with 1482 number of mutation $(\eta)$ having nucleotide diversity $(\pi)$ of $(\pi=0.3)$ were largely high on comparison with ChiLCB (MM-2) $(\pi>0.06)$ (Mishra et al. 2020). Therefore, the estimation suggests diverseness among populations. Furthermore, the number of haplotype (h) and haplotype diversity (Hd) was also estimated for the sequence datasets, which decipher the total number of haplotype (h) distribution for DNA-A was 32 and its haplotype diversity was detected equal to 1(1.000). Similarly, among its ORFs the haplotype diversity (Hd) were found close to 1 for gene pre-CP, REn, Rep, C4 and equal to 1 for gene $\mathrm{CP}$ and TrAP which support the relative contribution of genes in DNA polymorphism. Simultaneously, for betasatellite the total number of haplotype (h) and its diversity were detected 17 and equal to 1(1.000) respectively. Therefore, the estimation suggests the uniqueness within population and subpopulation (Table 5).

254 Neutrality tests were used to assess and understand the demographic selection acting on 255 genetic population of begomoviruses and associated satellite molecules. Tajima's D was 256 chosen for evaluation criteria which statistically reflect the negative Tajima's D value for 
258

259

260

261

262

263

values were negative, which indicates the large proportion of genetic segregation might be there within sequence datasets which were unique to individual sequences. Simultaneously, the other parameters such as Fu \& Li's $D$ and Fu \& Li's $F$ tests of population statistics were also evaluated resulting in negative values for DNA-A, its ORFs and betasatellites, indicating reiterating of purifying selection and population expansion which might be due to the inherent diversity. Nevertheless, the combination of Tajima's D, Fu \& Li's $D$, and Fu \& Li's $F$ negative values for DNA-A, its ORFs and associated satellite population revealed the conserved nature of the gene. Such evidences of nucleotide diversity might be expected when a selective sweep succeed the expansion of population and when most observable segregation functioning at the nucleotide level in a population are momentary and are eventually withdrawn by purifying selection (Table 6).

\section{Discussion}

India shares a large portion of the population which depends on small-area agricultural farming for their subsistence and income. A wide variety of diseases and their infection rate has been seen causing devastating effects both on crop yield and human persistence. Undoubtedly, the cultivation practices and presence of tropical climate conditions in the Indian subcontinent aids to the prevalence of a large number of plant viruses here, Papaya leaf curl virus (PaLCuV) found causing papaya leaf curl disease (PaLCuD), which affects plant growth, fruit size, quality and quantity, slowing its yield (Shahid et al. 2013; Varun et al. 2017) thus accelerating the spread of viral diseases. Additionally, climate change, adaptability and fast distribution of vectors and viruses is of major concern for the agriculture sector as they are greatly contributing to the Indian economy.

Across India, a full-length sequence of reported begomoviruses infecting Carica papaya were collected from NCBI and arranged into three specific datasets containing sequence of DNAA, its ORFs and associated betasatellites. The recurrent occurrence of recombination and nucleotide substitution alike to RNA viruses are mostly attributed factors to contribute high genetic variability among begomovirus populations which may significantly step up their evolution by expanding the combinations of pre-existing nucleotide segregation created by mutation (Duffy and Holmes 2008, 2009). Accordingly, recombination and mutation are often stated as the chief contributors to genetic variability which is the subject matter of investigation in the present study by the aid of molecular and computational efficacy. 
289 The results revealed partitioning-based diversification, recombination together with mean 290 substitution and purifying selection, as major contributor of observed levels of genetic 291 variability across begomovirus genomes. The phylogeny-based partitioning method 292 qualitatively estimated from the aligned whole-genome sequences and satellite molecules 293 showing mean branch length were found useful in quantifying the effect of recombination 294 event. To exclude any others biases that strengthen the significant differences between the 295 degrees of intra and inter-specific variability, we checked for different rate of transition and 296 transversion substation rate and transition /transversion bis(R) which supports the 297 contribution of mutation for nucleotide polymorphisms in a population (Mishra et al. 2020).

298 Previous studies have revealed that recombination happens at high frequencies in 299 begomovirus populations, which uses a conserved feature i.e., rolling circle mechanism for 300 replicating their genomes and make it mechanistically recombination-prone thus, generating 301 recombination breakpoints in a non-random location (Martin et al. 2011). Recombination 302 rates are threatened for plant viruses. Our experimental analysis detected a recombination 303 event in genomic region of datasets, showing variable parents which results in uneven 304 distribution of recombination breakpoints supporting genetic diversity. However, the 305 statistically measurable recombination rate seems to be lower than the mutation rate in 306 sequence datasets. Even in such case recombinational event act actively but consequently, the mutational dynamics were still the leading forces in shaping the standing genetic variability.

308 In other words, the relative contribution is potentially better than that estimated from our 309 studies.

310 The substantial aspects towards the population genetics are possibly accommodated by 311 mutation along with recombination, neutral selection, genetic drift and gene flow, which acts 312 significantly to shape the genetic structure of populations. Further, we used coalescent 313 Bayesian skyline model, strict and relaxed clock log normal and found relaxed clock as a 314 prior to explore genetic diversity. Additionally, it is important to address the key issue that 315 refers to the uneven distribution of the genetic variation across begomovirus genomes. In this context, the combination and pattern of various factors are responsible for affecting genetic variability through distribution of polymorphisms in non-random manner in the genomic regions of begomoviruses of datasets (Mishra et al. 2020).

319 Nevertheless, the standing genetic variability in all begomovirus populations was dominated 320 by mutation, since for all datasets nucleotide diversity( $\pi$ ) were found high, suggesting the 321 diverseness. Neutrality methods are used for finding positive selection to validate the 322 significance of positive or diversifying selection in shaping the uneven levels of genetic 
323 variability across genome (Lima et al. 2017). Based on analysis negative values for given

324 datasets indicates the reiterating of purifying selection and population expansion. Moreover, 325 not any sporadic cases of positively selected sites were spotted. Therefore, our results clearly 326 eliminate positive selection as a major contributor for the neutral selection in population. By 327 some mean purifying selections are found responsible in accelerating the genetic variability 328 in specific regions of genes.

329 Although the number of sequence data for DNA-A and betasatellites are small, and for 330 alphasatellite the database is insufficient, the results of the present study possibly provide 331 meaningful basic information that contributed greatly to diversification of begomoviruses 332 particularly causing papaya leaf curl diseases and acknowledged the evolutionary potential, 333 principally in the context of recombination, adaptation, genetic diversity, emergence and

\section{References}

Adiaha MS, Adiaha MS (2017) Effect of nutritional, medicinal and pharmacological 348 properties of papaya (Carica papaya L.) to human development: a review. World Sci News $349 \quad 2: 238-249$.

350 Brown JK, Fauquet CM, Briddon RW, Zerbini M, Moriones E, Navas Castillo J (2012) 351 Geminiviridae. In: King AMQ, Adams MJ, Carstens EB, Lefkowitz EJ (ed) In Virus 352 Taxonomy- Ninth Report of the International Committee on Taxonomy of Viruses. Elsevier 353 Academic Press., San Diego, CA: pp 351-373

354 Duffy S, Holmes EC (2008) Phylogenetic evidence for rapid rates of molecular evolution in 355 the single-stranded DNA begomovirus Tomato yellow leaf curl virus. J Virol 82:957-965. 356 https://doi.org/10.1128/JVI.01929-07

357 Duffy S, Holmes EC (2009) Validation of High Rates of Nucleotide Substitution in 358 Geminiviruses: Phylogenetic Evidence from East African Cassava Mosaic Viruses. J of Gen 359 Virol 90:1539-47. https://doi.org/10.1099/vir.0.009266-0

360 Evans EA, Ballen FH (2012) An Overview of Global Papaya Production, Trade, and 
361 Consumption. FE913.

362 FAOSTAT (2019) Food and Agriculture organization of the United Nation Database. 363 http://www.fao.org/faostat/en/\#data/QC. Accessed 26 June 2021.

364 Fiallo-Olivé E, Tovar R, Navas-Castillo J (2016) Deciphering the biology of deltasatellites 365 from the New World: maintenance by New World begomoviruses and whitefly transmission. 366 New Phytol 212:680-692. https://doi.org/10.1111/nph.14071

367 Fougat R S, Purohit AR, Kumar S, Parekh MJ, Kumar M (2015) SSR based genetic diversity 368 in Abelmoschus species. Indian J Agr Sci 85:1223-1228. https://doi.org/10.1007/s13562-016$369 \quad \underline{0378-2}$

370 Guo T, Guo Q, Cui X, Liu Y, Hu J, Liu S (2015) Comparison of transmission of papaya leaf 371 curl China virus among four cryptic species of the whitefly Bemisia tabaci complex. Sci Rep 372 25:15432. https://doi.org/10.1038/srep15432

373 Hanley-Bowdoin L, Bejarano ER, Robertson D, Mansoor S (2013) Geminiviruses: masters at 374 redirecting and reprogramming plant processes. Nat Rev Microbiol 11:777-788. 375 https://doi.org/10.1038/nrmicro3117

376 Jenkins GM, Rambaut A, Pybus OG, Holmes EC (2002) Rates of molecular evolution in 377 RNA viruses: a quantitative phylogenetic analysis. J Mol Evol 54:156-165. 378 https://doi.org/10.1007/s00239-001-0064-3

379 Kumar RV, Singh AK, Singh AK, Yadav T, Basu S, Kushwaha N, Chattopadhyay B, 380 Chakraborty S (2015) Complexity of begomovirus and betasatellite populations associated 381 with chilli leaf curl disease in India. J Gen Virol 96(10):3143-3158. 382 https://doi.org/10.1099/jgv.0.000254

383 Kumar S, Stecher G, Li M, Knyaz C, Tamura K (2018) MEGA X: Molecular Evolutionary 384 Genetics Analysis across Computing Platforms. Mol Biol Evol 35:1547-1549. 385 https://doi.org/10.1093/molbev/msy096

386 Lima ATM, Silva JCF, Silva FN, Castillo-Urquiza GP, Silva FF, Seah YM (2017) The 387 diversification of begomovirus populations is predominantly driven by mutational dynamics. 388 Virus Evol 3:vex005. https://doi.org/10.1093/ve/vex005

389 Lozano G, Trenado HP, Fiallo-Olivé E, Chirinos D, Geraud-Pouey F, Briddon RW (2016) 390 Characterization of non - coding DNA satellites associated with sweepoviruses (genus 391 Begomovirus, Geminiviridae) - definition of a distinct class of begomovirus - associated 392 satellite. Frontiers Microbiology 7:162. https://doi.org/10.3389/fmicb.2016.00162

393 Martin DP, Lefeuvre P, Varsani A et al (2011) Complex Recombination Patterns Arising 394 During Geminivirus Coinfections Preserve and Demarcate Biologically Important Intra395 Genome Interaction Networks. PloS Pathogens 7:e1002203. 396 https://doi.org/10.1371/journal.ppat.1002203

397 Martin DP, Murrell B, Golden M, Khoosal A, Muhire B (2015) RDP4: Detection and 398 analysis of recombination patterns in virus genomes. Virus Evol 1:vev003. 399 https://doi.org/10.1093/ve/vev003 
400 Marwal A, Nehra C, Verma RK, Mishra M, Srivastava D, Choudhary P, Gaur RK 401 (2021) First report of papaya leaf curl virus and its associated papaya leaf curl betasatellite 402 infecting Catharanthus roseus plants in India. The Journal of Horticultural Science 403 Biotechnology https://doi.org/10.1080/14620316/2021/1912646

404 Mishra M, Verma RK, Marwal A, Sharma P, Gaur RK (2020) Biology and Interaction of the 405 Natural Occurrence of Distinct Monopartite Begomoviruses Associated with Satellites 406 in Capsicum annum from India. Frontiers Microbiology 11:512957. 407 https://doi.org/10.3389/fmicb.2020.512957

408 Moreno-Delafuente A, Garzo E, Moreno A, Fereres A (2013) A plant virus manipulates the 409 behavior of its whitefly vector to enhance its transmission efficiency and spread. PloS one 410 8(4):e61543. https://doi.org/10.1371/journal.pone.0061543

411 Nascimento AKQ, Lima JAA, Nascimento ALL, Beserra EAJr (2010) Biological, physical, 412 and molecular properties of a Papaya lethal yellowing virus isolate. Plant Disease 413 94(10):1206-1212. https://doi.org/10.1094/PDIS-11-09-0733

414 Nawaz-ul-Rehman MS, Fauquet CM (2009) Evolution of geminiviruses and their satellites. 415 FEBS letters 583(12):1825-1832. https://doi.org/10.1016/j.febslet.2009.05.045

416 Nehra C, Marwal A, Verma RK, Mishra M, Sharma P, Gaur RK (2019) Papaya yellow Leaf 417 curl virus: A newly identified Begomovirus infecting Carica papaya L. from the Indian 418 Subcontinent. The Journal of Horticultural Science and Biotechnology. 419 https://doi.org/10.1080/14620316.2019.1570827

420 Rambaut A, Drummond AJ, Xie D, Baele G, Suchard MA (2018) Posterior Summarization in 421 Bayesian Phylogenetics Using Tracer 1.7. Syst Biol 67(5):901-904. 422 https://doi.org/10.1093/sysbio/syy032

423 Rozas J, Ferrer-Mata A, Sánchez-DelBarrio JC, Guirao-Rico S, Librado P, Ramos-Onsins SE 424 (2017) DnaSP 6: DNA sequence polymorphism analysis of large data sets. Mol Biol Evol 425 34:3299-3302. https://doi.org/10.1093/molbev/msx248

426 Saxena S, Hallan V, Singh BP, Sane PV (1998) Nucleotide sequence and inter geminiviral 427 homologies of the DNA A of Papaya leaf curl geminivirus from India. Biochemistry and 428 Molecular Biology International 45:101-113. https://doi.org/10.1080/15216549800202472

429 Shahid MS, Yoshida S, Khatri-Chhetri GB, Briddon RW, Natsuaki KT (2013) Complete 430 nucleotide sequence of a monopartite Begomovirus and associated satellites infecting Carica 431 papaya in Nepal. Virus Genes 46(3):581-4. https://doi.org/10.1007/s11262-013-0888-0

432 Stanley J, Bisaro DM, Briddon RW, Brown JK, Fauquet CM, Harrison BD, Rybicki EP, 433 Stenger DC (2005) Geminiviridae. In: Fauquet CM, Mayo MA, Maniloff J, Desselberger U, 434 Ball LA (ed) VIIIth Report of the International Committee on Taxonomy of Viruses. Virus 435 Taxonomy. Elsevier/Academic Press, London, pp 1163-1169

436 Suchard MA, Lemey P, Baele G, Ayres DL, Drummond AJ, Rambaut A (2018) Bayesian 437 phylogenetic and phylodynamic data integration using BEAST 1.10. Virus Evol 4(1):vey016. 438 https://doi.org/10.1093/ve/vey016 
439 Urgessa OE, Itana DD, Raga OT (2019) Extraction of Papain from Papaya (Carica Papaya 440 L.) Fruit Latex and Its Application in Transforming Tannery Raw Trimming. Ethiopian 441 Journal of Science and Sustainable Development 6(2):22442 32. https://doi.org/10.20372/ejssdastu:v6.i2.2019.92

443 Usha R (1980) Characterization, diagnosis \& management of plantviruses. In: Rao GP, 444 Kumar PL, Holguı́n-Peña RL (ed) Studium Press, Houston, pp 387-392

445 Varun P, Ranade SA, Saxena S (2017) A molecular insight into papaya leaf curl - a severe 446 viral disease. Protoplasma 254 (6):2055-2070. https://doi.org/10.1007/s00709-017-1126-8

447 Varun P, Saxena S (2018) Association of tomato leaf curl Gujarat virus and tomato leaf curl 448 Bangladesh betasatellite on Papaya showing typical leaf curl symptoms in North India. 449 3Biotech 8:243. https://doi.org/10.1007/s13205-018-1254-7

450 Vij T, Prashar Y (2015) A review on medicinal properties of Carica papaya Linn. Asian 451 Pacific Journal of Tropical Disease 5(1):1-6. https://doi.org/10.1016/S2222-1808(14)60617-4

452 Yadav J, Yadav S, Mishra S (2015) Experimental evidences showing nutritional and 453 medicinal property of Carica papaya plant. International journal of science and research 454 6(12):21-25. https://doi.org/10.21275/ART20178569

Zerbini, F.M., Briddon, R.W., Idris, A., Martin, D.P., Moriones, E., Navas-Castillo, J., 456 Rivera-Bustamante, R., Roumagnac, P., Varsani, A., 2017. ICTV report consortium. ICTV 457 virus taxonomy profile: geminiviridae. J. Gen. Virol. 98 (2), 131-133. 458 https://doi.org/10.1099/jgv.0.000738. 


\section{Legend for Figures and Tables}

474 Fig1. Distribution of distinct begomoviruses isolates and associated satellites in India causing leaf 475 curl disease of Carica papaya (Follow Table 1).

476 Fig 2 . Maximum-likelihood phylogeny-based partitioning tree associated with papaya leaf curl 477 disease in India aligned using CLUSTAL W within MEGA v.10. (a) DNA-A; (b) Betasatellites.

479 Table 1. Features of begomoviruses causing leaf curl disease in papaya are identified in India.

480 Table 2. Substitution rate for begomoviruses and betasatellites associated with PaLCuD in 481 India.

Table 3 (a) Putative recombination events detected among begomoviruses associated with 484 papaya leaf curl disease, based on provided datasets from India; DNA-A and associated 485 betasatellite.

486 Table 3 (b) Putative recombination events detected among begomoviruses associated with 487 papaya leaf curl disease, based on provided datasets from India; six genes/ORF's of DNA-A.

$488 \mathrm{~b}$ Mean substitution and codon degeneracy rates for PaLCuD associated begomoviruses.

489 Table 5. Genetic diversity of begomoviruses and betasatellites associated with PaLCuD in 490 India.

491 Table 6. Different neutrality tests for the datasets obtained from identified begomoviruses 492 causing PaLCuD in India. 


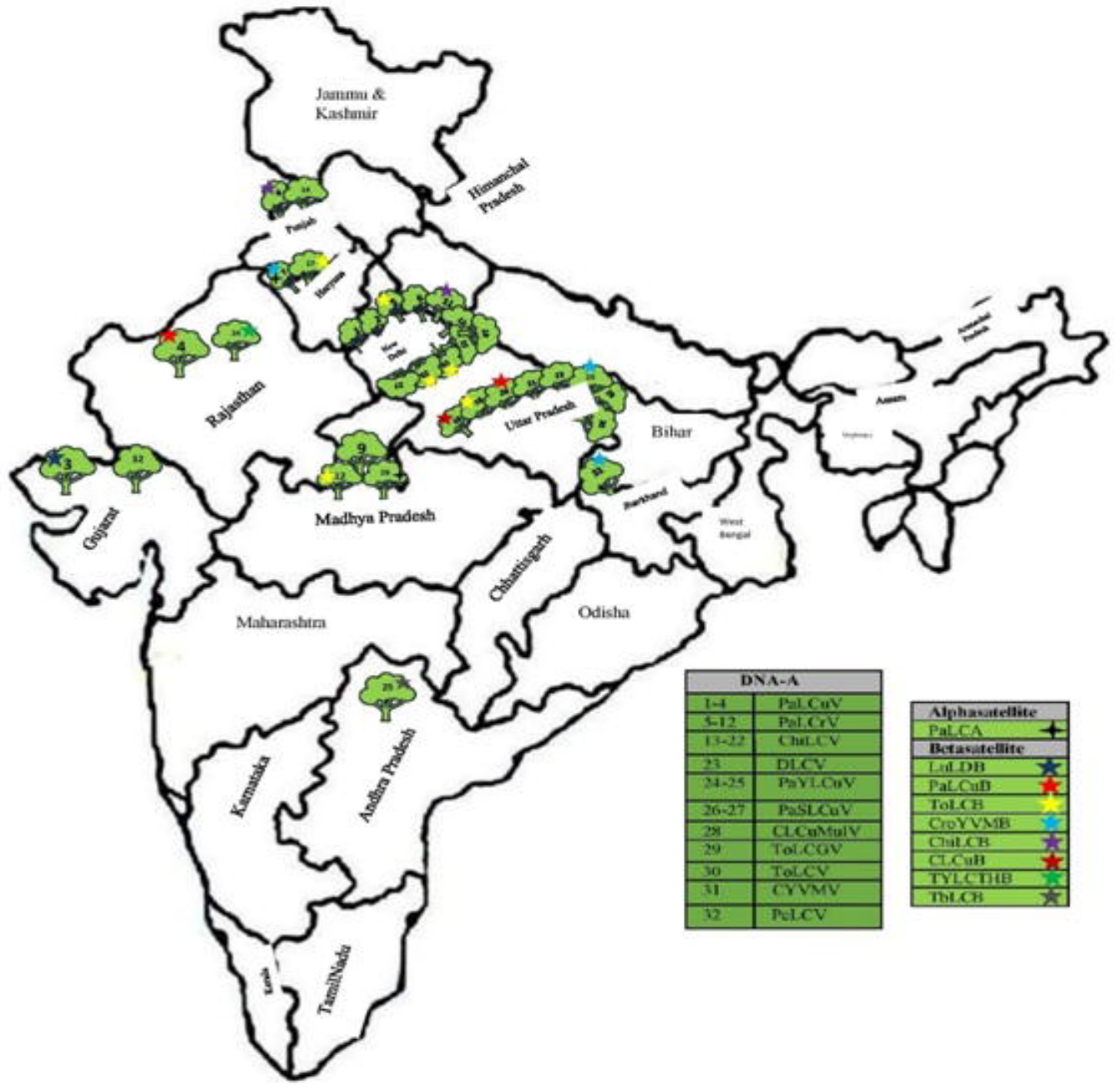




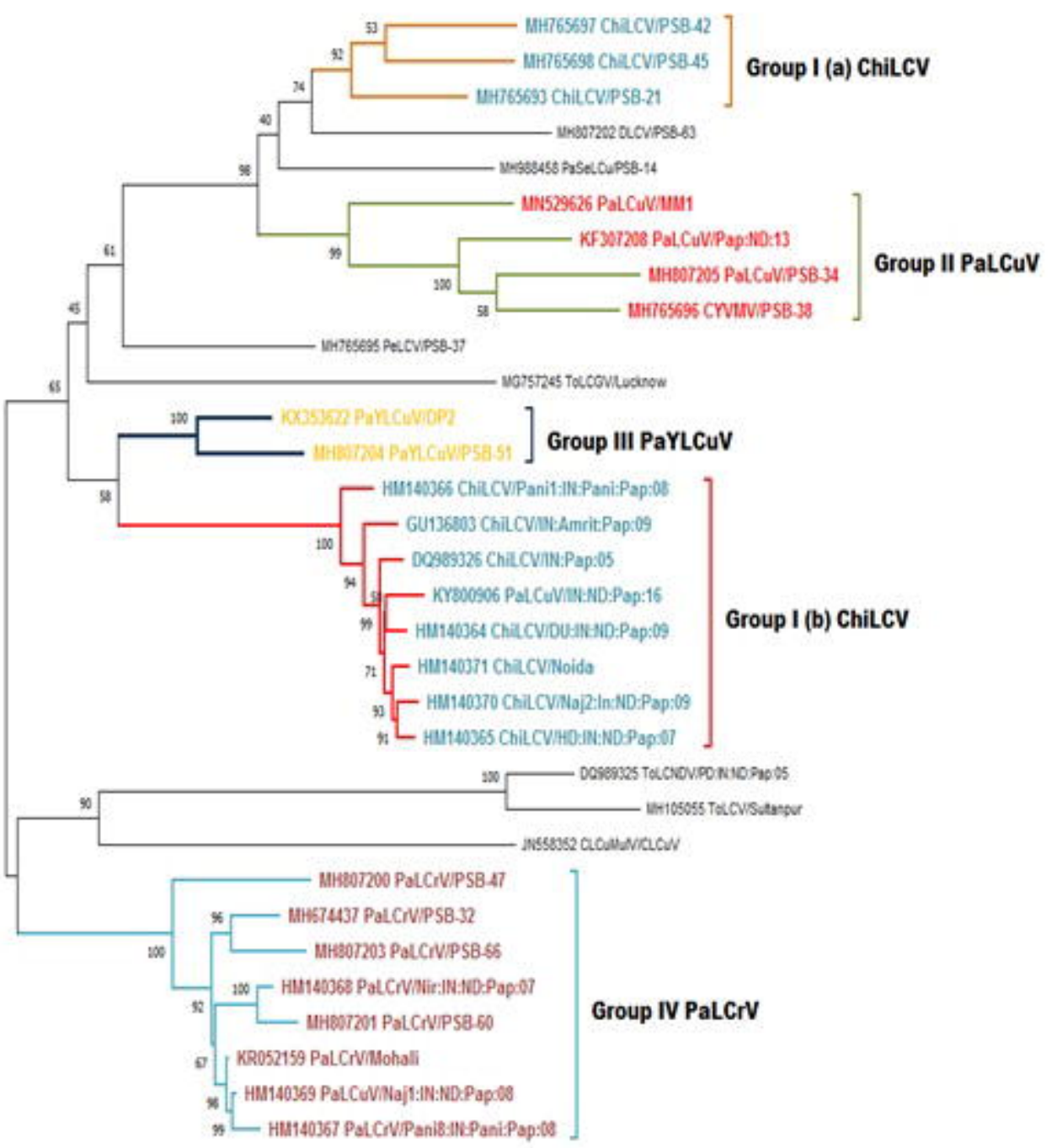




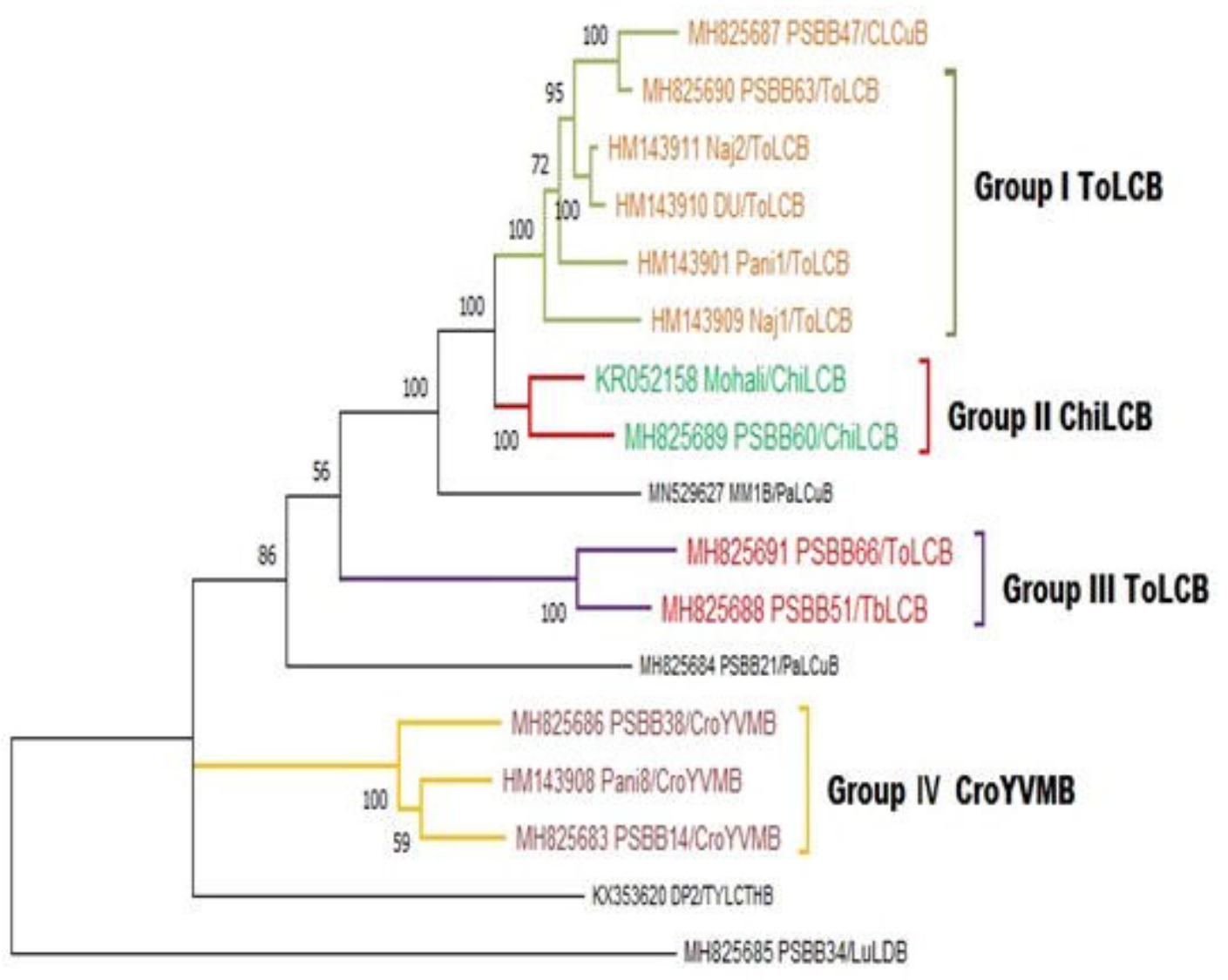

